\title{
SITUACIÓN DE LA CALIDAD DE AGUA PARA CONSUMO EN HOGARES DE NIÑOS MENORES DE CINCO AÑOS EN PERÚ, 2007-2010
}

\author{
Marianella Miranda ${ }^{1, a}$, Adolfo Aramburú1,a, Jorge Junco ${ }^{2, b}$, Miguel Campos 3 ,c
}

\begin{abstract}
RESUMEN
Objetivo. Estimar la proporción de niños menores de cinco años con acceso a agua de calidad y su comportamiento en función de la localización geográfica, abastecimiento de agua y situación de pobreza. Materiales y métodos. Encuesta continua (transversal repetida), por muestreo aleatorio multietápico, del universo de niños menores de cinco años residentes en el Perú. Se evaluó la presencia de cloro libre en muestras de agua para consumo en los hogares de 3570 niños (Lima metropolitana 666 , resto de costa 755 , sierra urbana 703, sierra rural 667 y selva 779 ). Se evaluó la presencia de coliformes totales y E. coli en muestras de agua de 2310 hogares (Lima metropolitana 445, resto de costa 510, sierra urbana 479, sierra rural 393 y selva 483). Resultados. La proporción nacional de niños menores de cinco años que residen en hogares con cloro libre adecuado en el agua para consumo, alcanza a 19,5\% del total, mientras que la correspondiente a agua libre de coliformes y E. coli asciende a 38,3\%. Existe una marcada diferencia de los resultados por área de residencia (los ámbitos más afectados fueron sierra rural y selva), red pública domiciliaria dentro de la vivienda y quintiles de ingreso. Conclusión. Existe una gran desventaja en los niños menores de cinco años provenientes de hogares pertenecientes al área rural y en extrema pobreza, para acceder al consumo de agua de calidad. Esta situación representa un serio problema para el control de las enfermedades diarreicas y la desnutrición infantil.
\end{abstract}

Palabras clave: Microbiología del agua; Calidad del agua; Abastecimiento de agua; Encuestas nutricionales; Pobreza; Perú (fuente: DeCS BIREME).

\section{STATE OF THE QUALITY OF DRINKING WATER IN HOUSEHOLDS IN CHILDREN UNDER FIVE YEARS IN PERU, 2007-2010}

\begin{abstract}
Objective. To evaluate the proportion of children under five with access to quality water and its behavior according to geographical location, water supply and poverty status. Material and methods. Continuous survey (repeated cross), with multistage random sampling, being the universe children under five years living in Peru. We evaluated the presence of free chlorine in drinking water samples in the dwellings of 3570 children (Metropolitan Lima 666, Rest of Coast 755, Urban Sierra 703, Rural Sierra 667, and Jungle 779). We evaluated the presence of total coliforms and $E$. coli in water samples of 2310 households (445 Metropolitan Lima, Rest of Coast 510, Urban Sierra 479, Rural Sierra Selva 393 and 483). Results. The national proportion of children under five years living in households with adequate free chlorine in drinking water reaches $19.5 \%$ of the total, while water free of coliforms and $E$. coli is $38.3 \%$. There is a marked difference in results by area of residence (the most affected areas were rural Sierra and Jungle), public network at home inside the dwelling and income quintiles. Conclusion. Children under five years living in households belonging to the rural areas and extreme poverty, have a great disadvantage to access quality water consumption. This situation represents a serious problem for the control of diarrheal diseases and children malnutrition.
\end{abstract}

Key words: Water microbiology; Water quality; Water supply; Nutrition surveys; Poverty; Peru (source: MeSH NLM).

\section{INTRODUCCIÓN}

El Gobierno Peruano ha enfatizado estrategias de intervención articulada y políticas nacionales, que conlleven a la reducción de la desnutrición ${ }^{(1,2)}$, en coherencia con políticas internacionales como los
Objetivos de Desarrollo del Milenio, que incluyen Reducir a la mitad, para el año 2015, el porcentaje de personas que carezcan de acceso sostenible a agua potable ${ }^{(3)}$.

El acceso de la población al agua de calidad es un derecho ${ }^{(4)}$. La mejora del saneamiento básico de

\footnotetext{
Dirección Ejecutiva de Vigilancia Alimentaria y Nutricional, Centro Nacional de Alimentación y Nutrición, Instituto Nacional de Salud, Lima, Perú.

Asesoría en Nutrición y Salud - ASENSA SAC, Lima, Perú.

3 Departamento de Física, Informática y Matemáticas, Facultad de Ciencias y Filosofía, Universidad Peruana Cayetano Heredia. Lima, Perú.

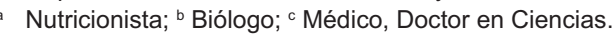

Recibido: 28-09-10 Aprobado: 07-12-10 
la vivienda reduce la incidencia de enfermedades infecciosas entre 20 y $80 \%{ }^{(5,6)}$.

Para el año 2009, el abastecimiento por red pública dentro de la vivienda en el Perú, aumentó en 5,9\% con relación a lo registrado en el año 2000. La disponibilidad de desagüe por red pública dentro de la vivienda en el 2009 solo alcanzó al $51,6 \%$ de la población, siendo $3,5 \%$ más que el $2000{ }^{(7)}$.

Los países de América Latina no han podido llegar a una cobertura total en abastecimiento y saneamiento de agua. El problema es aun más serio en calidad del agua y protección del recurso hídrico ${ }^{(8)}$.

Por tanto, indicadores como agua con cloro libre adecuado y ausencia de coliformes y E. coli son elementos claves para la vigilar la calidad del agua ${ }^{(9)}$. El presente artículo presenta la situación de dichos indicadores, con datos del Monitoreo Nacional de Indicadores Nutricionales (MONIN) 2007-2010, ejecutado por el Centro Nacional de Alimentación y Nutrición, del Instituto Nacional de Salud (INS/CENAN).

\section{MATERIALES Y MÉTODOS}

\section{TIPO DE ESTUDIO}

EI INS/CENAN, órgano de línea técnico normativo del Instituto Nacional de Salud, encargado de conducir el sistema de vigilancia nutricional en el Perú (10) ha llevado a cabo el MONIN 2007-2010 (11). Su objetivo ha sido medir la tendencia en la frecuencia y los factores de la desnutrición infantil, así como las coberturas de los programas de intervención. Su método ha sido una encuesta continua (transversal repetida), por muestreo aleatorio multietápico, del universo de niños menores de cinco años y gestantes residentes en el Perú, dividido en cinco ámbitos geográficos y seis periodos aproximadamente trimestrales (semanas permutadas aleatoriamente que fueron $78 \%$ del tiempo entre el 03 de noviembre de 2007 al 02 de abril de 2010).

\section{POBLACIÓN Y MUESTRA}

La muestra total del MONIN comprende 3756 niños menores de cinco años. Para el presente artículo, se ha incluido la evaluación de cloro en muestras de agua correspondiente a 3570 hogares, distribuidos por ámbitos de la siguiente manera: Lima metropolitana 666; resto de costa 755; sierra urbana 703; sierra rural 667 y selva 779. Asimismo, desde noviembre de 2008 se ha evaluado la presencia de coliformes totales y $E$. coli en 2310 hogares, según la siguiente distribución: Lima
Metropolitana 445; resto de costa 510; sierra urbana 479; sierra rural 393 y selva 483.

El cálculo del tamaño muestral del MONIN, mediante la fórmula de estimación de proporciones con efecto de diseño ${ }^{(12)}$, obtuvo un tamaño neto aproximado de 120 , necesarios para obtener un margen de error $(95 \%$ de confianza) de aproximadamente 7,5 a $15,0 \%$ para rangos de proporción de prevalencia entre 20 a $80 \%$, con un efecto de diseño de 1,5 en cualquier estrato deseado. Para el presente artículo se incluyeron todos los sujetos con datos disponibles para las variables medidas.

El universo principal fue dividido en cinco estratos: Lima Metropolitana (la provincia de Lima y la provincia constitucional del Callao), resto de costa (centros poblados de la vertiente occidental de los andes por debajo de los $2300 \mathrm{~m}$ de altitud), sierra urbana (centros poblados a $2300 \mathrm{~m}$ de altitud o más, con poblaciones de 2000 habitantes o más), sierra rural (centros poblados a $2000 \mathrm{~m}$ de altitud o más, con poblaciones menores a 2000 habitantes) y selva (centros poblados de la vertiente oriental de los Andes por debajo de los 2500 $\mathrm{m}$ de altitud) ${ }^{(13)}$.

\section{PROCEDIMIENTOS}

Para este análisis secundario se incluyó las siguientes variables: lugar de procedencia de la muestra de agua, fecha, hora y temperatura de incubación, presencia o ausencia de coliformes y E. coli, recojo de muestra de agua para cloro y tipo de reacción al kit. Los niveles de pobreza fueron estimados según la clasificación distrital de quintiles de ingreso, que van del nivel I que es el más pobre al $\mathrm{V}$, menos pobre ${ }^{(14)}$.

Los procedimientos para la recolección de la muestra y análisis descritos a continuación se encuentran en los protocolos del Manual del Encuestador Bioquímico del INS/CENAN ${ }^{(15) .}$

Determinación de cloro residual en agua. En cada hogar evaluado, el encuestador proporcionó a la entrevistada un vaso descartable y le solicitó le trajera una muestra del "Agua que utiliza la familia para lavar o preparar sus alimentos". Con el agua proporcionada se realizó un doble enjuague del vial del kit, en el cual se separó $5 \mathrm{~mL}$ de dicha muestra y se añadió cuatro gotas del reactivo Chlorine Test ${ }^{\circledR}{ }^{(16)}$, homogeneizando con movimientos circulares. El reactivo permite un análisis semicuantitativo de cloro residual, basado en la reacción del cloro con la tetrametilbenzidina (TMB), que produce una coloración amarilla de diferente intensidad, que se comparó con las escalas de color incluidas en el 
kit ( $0 ; 0,1 ; 0,25 ; 0,5 ; 1$ o $2 \mathrm{mg} / \mathrm{L}$ de cloro), y se anotó la escala correspondiente en el formulario de encuesta.

\section{Determinación de la presencia de coliformes totales} y E. coli. Esta prueba se realizó a partir del periodo 2008-IV (03-nov-2008). Para ello, se utilizó el kit Colilert ${ }^{\circledR}{ }^{(17)}$, el cual se basa en la detección de dos enzimas ( $\beta$-galactosidasa y $\beta$-glucuronidasa) mediante fluorescencia. A partir del periodo 2009-Il (13-abr-2009), se utilizó el kit Readycult ${ }^{\circledR}{ }^{(18)}$, el cual incorpora una prueba confirmatoria de $E$. coli, mediante la reacción de Kovacs o prueba de indol.

Para ambos reactivos, en cada hogar, el encuestador obtuvo una muestra de $100 \mathrm{~mL}$ del agua utilizada por la familia para preparar sus alimentos. Para las muestras provenientes del caño, se tuvo la precaución de desinfectar con alcohol, flamear y dejar correr el agua por un espacio de dos minutos para evitar cualquier residuo. Para las muestras provenientes de un recipiente de depósito, se usó un vaso descartable limpio a una profundidad de por lo menos $20 \mathrm{~cm}$, cuidando no tocar el fondo del recipiente.

La muestra de agua fue almacenada dentro de un frasco de polietileno con tiosulfato de sodio como agente declorinante, e inmediatamente refrigerada dentro de un conservador portátil (cooler) con unidades de hielo gel congeladas, hasta ser incubadas en un plazo no mayor de 30 horas. Los lugares de incubación fueron los laboratorios regionales (hospitales, establecimientos de salud) cercanos a las zonas de estudio o en el laboratorio del CENAN, dependiendo de la disponibilidad de una incubadora que mantenga una temperatura de $35 \pm 0,5{ }^{\circ} \mathrm{C}$.

Antes de colocar en la incubadora, se le agregó a cada muestra el contenido de un cojín de reactivo (Collilert $\AA$ ) o Readycult $\circledR$ ) y se incubó durante 24 horas. Se analizó el viraje de color para evaluar la presencia de coliformes totales; la coloración amarilla (Collilert $\AA$ ) o azul verdoso (Readycult $\AA$ ) indicaban la presencia de coliformes totales. E. coli se evaluó mediante la presencia de fluorescencia a la luz UV a $365 \mathrm{~nm}$ de longitud de onda y $15 \mathrm{~cm}$ de distancia. A partir del periodo 2009-II, a las muestras positivas a fluorescencia se les agregó 1 $\mathrm{mL}$ de reactivo de Kovacs como prueba confirmatoria; la presencia de un anillo de color rojizo confirma la presencia de E. coli.

Para el análisis de datos, se definió agua con cloro libre adecuado al resultado igual al nivel $0,5 \mathrm{mg} / \mathrm{L}$ del test de cloro y agua con ausencia de coliformes y $E$. coli, a la reacción negativa a las evaluaciones de dichos gérmenes ${ }^{(19)}$.

\section{ANALISIS ESTADÍSTICO}

La información fue analizada mediante el paquete estadístico SPSS ${ }$, v. 15.0 con módulo Complex Samples (20), con el cual se obtuvieron estimados ponderados de prevalencia y sus errores estándar ajustados al diseño muestral.

\section{RESULTADOS}

La proporción nacional de niños menores de cinco año en hogares con agua con cloro libre adecuado alcanza a 19,5\% (IC95\%: 15,9-23,1\%), mientras que la correspondiente a agua libre de coliformes y $E$. coli es de $38,1 \%$ (IC95\%: 33,6-42,9\%).

Se evidencia asimismo, una marcada diferencia entre el área geográfica urbana y rural del país (Figura 1). La proporción de niños en hogares con agua con cloro libre adecuado en el área urbana es de 24,2\% (IC95\%: 19,8$28,5 \%)$ y en el área rural es de 0,5\%(IC95:\% 0,0-1,4\%). Con respecto a la disposición de agua con ausencia de coliformes y $E$. coli, dicha proporción alcanza a $44,4 \%$ (IC95\%: 39,1-49,8\%) en el área urbana y 4,9\% (IC95\%: $1,5-8,2)$ en la zona rural.

La proporción de niños menores de cinco años en hogares con agua con cloro libre adecuado y ausencia de coliformes y E. coli, varía por ámbitos geográficos, evidenciando una marcada desventaja para la sierra rural y selva del país (Figura 2).

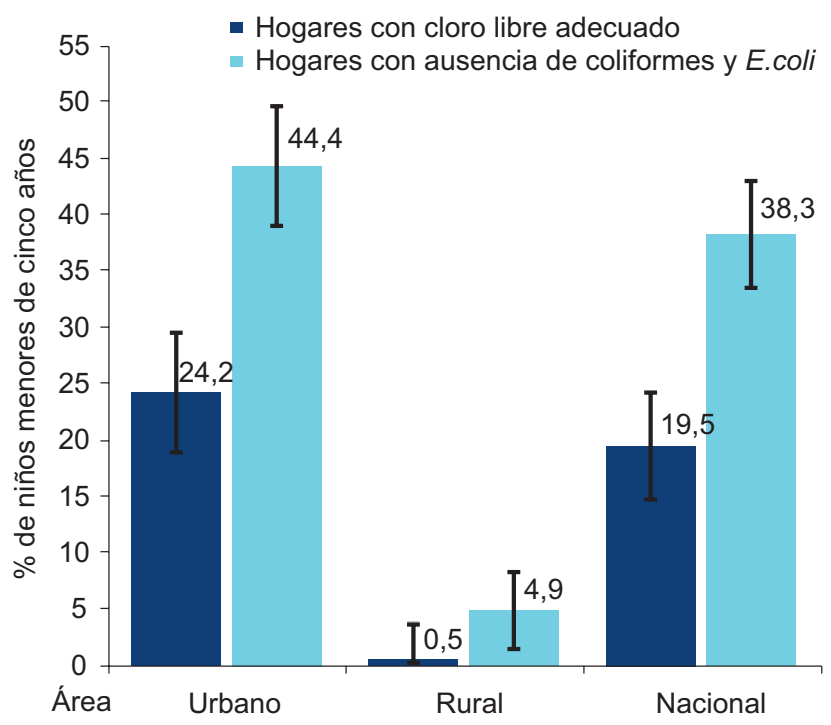

Figura 1. Indicadores de calidad de agua para consumo, según área geográfica en hogares de niños menores de cinco años, Perú 2007-2010. 


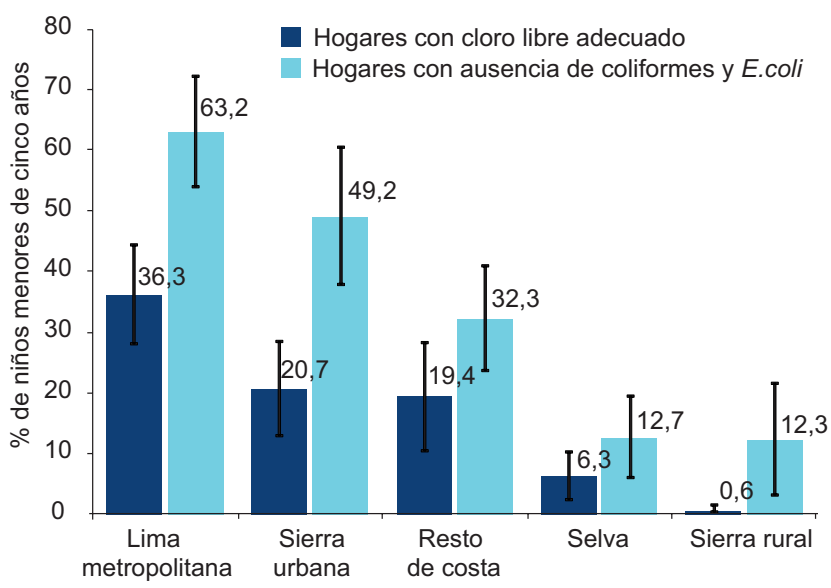

Figura 2. Indicadores de calidad de agua para consumo, según ámbitos geográficos en hogares de niños menores de cinco años, Perú 2007-2010.

La proporción de niños menores de cinco años en hogares con cloro libre adecuado y ausencia de coliformes y $E$. coli, varía según la fuente abastecimiento (Figura 3). Así, cuando el agua provino de la red pública dentro de la vivienda, el cloro libre adecuado estuvo presente en 30,2\% (IC95\%: 25,2-35,1\%); mientras que en el agua de otra procedencia, estuvo presente en el 5,4\% (IC95\%: 2,7- 8,1\%). Con respecto a la ausencia de coliformes y E. coli, la mayor proporción se encontró cuando el agua provino de la red pública dentro de la vivienda (52,6\%; IC95\%: 46,5- 58,7\%), en comparación con los que procedían de otra fuente $(16,6 \%$; IC95\%: 11,7- 21,4\%).

Al evaluar la calidad de agua para consumo según el nivel de pobreza, podemos apreciar, tanto para cloro libre adecuado, como para ausencia de coliformes y $E$. coli, un deterioro de las cifras mientras más pobres son los hogares (Figura 4).

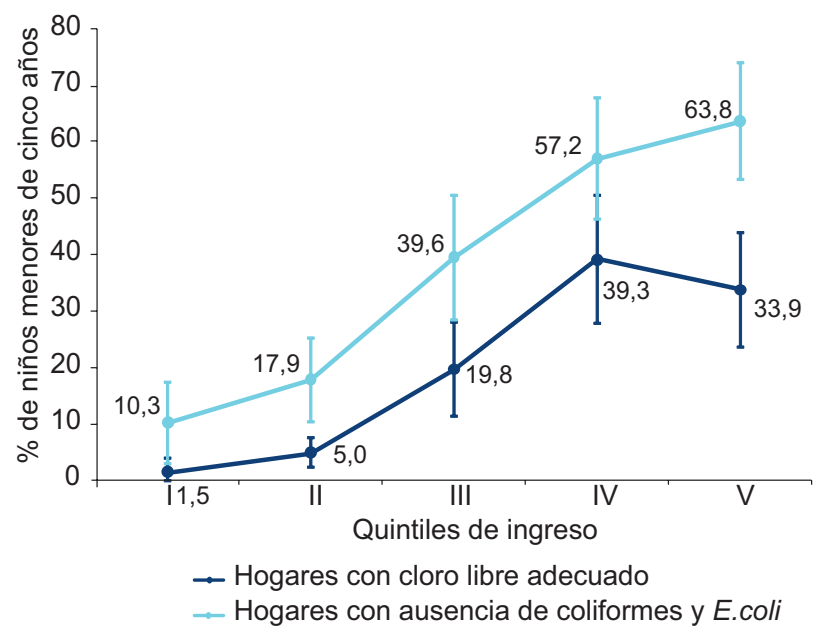

Figura 4. Indicadores de calidad de agua para consumo, según quintiles de ingreso en hogares de niños menores de cinco años, Perú 2007-2010.

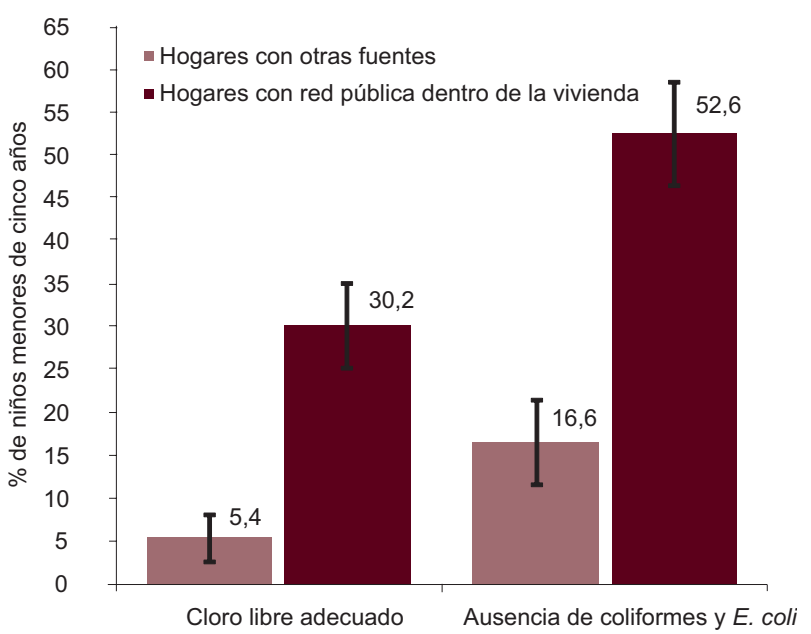

Figura 3. Indicadores de calidad de agua para consumo, según fuente de abastecimiento en hogares de niños menores de cinco años, Perú 2007-2010.

En relación con la evolución de los indicadores en el tiempo (Figura 5), podemos apreciar que el cloro libre adecuado muestra una ligera reducción durante el periodo de estudio. Sin embargo, el comportamiento observado se acompaña de una acentuada reducción del indicador de ausencia de coliformes y $E$. coli.

\section{DISCUSIÓN}

Los resultados indican que a nivel nacional, aun los hogares con red pública dentro de la vivienda y del quintil más alto de ingresos, tienen problemas en la calidad del agua para consumo, pues presentan cloro libre inadecuado y elevada presencia de coliformes y E. coli. Es evidente la marcada desigualdad en estos indicadores en el área rural, ámbitos de sierra y selva, y los quintiles de ingreso más pobres.

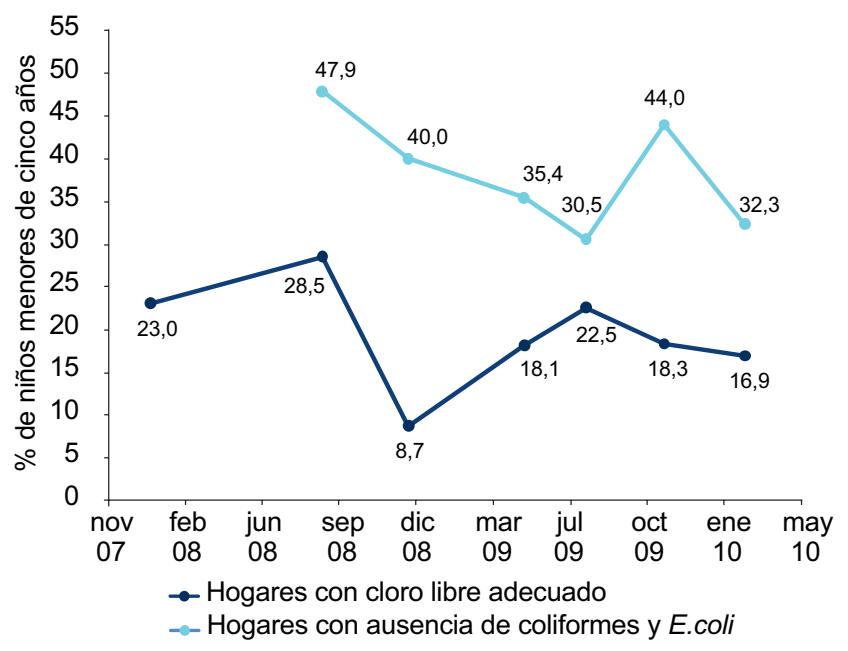

Figura 5. Comportamiento de los indicadores de calidad de agua para consumo en el tiempo en hogares de niños menores de cinco años, Perú 2007-2010. 
Aunque el protocolo puede haber tenido pequeñas variaciones en algún punto de la recolección de la muestra, en lo relacionado con métodos recomendados (19) y la unidad niño en el universo MONIN puede generar algunas diferencias de valor numérico, la situación de los indicadores mostrados no parece ser explicada por la metodología. Si hubo modificaciones en las condiciones de medición de campo, esperaríamos una subestimación de la presencia de coliformes y E. coli, dado que el periodo de refrigeración pudo durar entre 24 y 30 horas en algunos casos y una sobreestimación por no haber incluido un drenaje previo a chorro por dos minutos (19). Desconocemos si el uso de vasos descartables sin enjuague previo ${ }^{(19)}$ y llenados por la madre afectan la determinación de cloro libre residual.

Observaciones recientes en diversos lugares del Perú (19,21-27) son consistentes con estos hallazgos. Las causas de los problemas encontrados sobre la calidad de agua para consumo podrían ser explicados, sin ser excluyentes, por la insuficiente cobertura de las redes, cuando estas no mantienen cantidad o calidad constante o cuando en las conexiones, transporte o almacenamiento se anula el efecto del cloro o se introduce contaminación (19). A pesar del aumento de cobertura de agua potable en el Perú (28), los datos resaltan lo crítico que es prestar atención a las otras causas. Debe notarse que el MONIN no ha efectuado las determinaciones en las redes sino en el hogar, que, aunque se le considere excepcional como punto de muestreo ${ }^{(19)}$, es en realidad el punto principal de uso donde se decide el efecto final de los sistemas de aprovisionamiento de agua. Los indicadores del MONIN tampoco han abarcado todo el concepto de agua segura ${ }^{(29)}$.

EI MONIN incluyó los indicadores descritos de calidad de agua para consumo, por ser este un factor determinante reconocido para las enfermedades transmisibles ${ }^{(30)}$, las cuales son causas inmediatas de la desnutrición infantil (5). La situación insatisfactoria de la calidad de agua para consumo hallada en el presente estudio, afecta el séptimo Objetivo de Desarrollo del Milenio ${ }^{(3)}$ y las metas nacionales para la desnutrición ${ }^{(31)}$. El abastecimiento de agua tiene una interacción estrecha con la pobreza ${ }^{(32)}$, otro determinante clave de la desnutrición. El Estado peruano, en todos sus niveles, tiene la responsabilidad de mejorar la calidad de agua para consumo de la población.

\section{Fuentes de Financiamiento}

La ejecución ha sido financiada por el INS/CENAN. EI diseño y análisis se han financiado con fondos de INS/ CENAN, USAID, Banco Mundial, Banco Interamericano del Desarrollo, PARSALUD y Ministerio de Economía y Finanzas del Perú.

\section{Conflictos de Interés}

Los autores declaran no tener conflictos de interés en la publicación de este artículo.

\section{REFERENCIAS BIBLIOGRÁFICAS}

1. Perú, Presidencia del Consejo de Ministros. DS 0802007-PCM: Aprueban Plan de Operaciones de la Estrategia Nacional CRECER. Lima: PCM; 2007.

2. Perú, Presidencia del Consejo de Ministros. Decreto Supremo 032-2010-PCM Aprueba la incorporación de 239 distritos en la tercera etapa de implementación de la Estrategia Nacional CRECER, haciendo un total de 1119 distritos priorizados. Lima: PCM; 2010.

3. Naciones Unidas. Objetivos de desarrollo del milenio. Informe 2010. Ginebra: ONU; 2010.

4. World Health Organization. The right to water. Geneva: WHO; 2003.

5. Fondo de las Naciones Unidas para la Infancia. Estado mundial de la infancia 1998. Nueva York: UNICEF; 1998.

6. Heller L. Saneamiento y salud. Brasilia: OPS; 1997.

7. Instituto Nacional de Estadística e Informática. Perú - Encuesta Demográfica y de Salud Familiar - ENDES Continua 2009 Informe Principal. Lima: INEl; 2010.

8. Organización Panamericana de la Salud. Informe regional sobre la evaluación 2000 en la Región de las Américas: agua potable y saneamiento, estado actual y perspectivas. Washington DC: OPS; 2001.

9. Organización Mundial de la Salud. Guías para la calidad del agua potable. $3^{\text {ra }}$ ed. Ginebra: OMS; 2006.

10. Perú, Presidencia del Consejo de Ministros. DS 0342002-PCM: Fusionan diversos programas de apoyo alimentario y nutricional bajo la administración del PRONAA. Lima: PCM; 2002.

11. Instituto Nacional de Salud. Monitoreo Nacional de Indicadores Nutricionales (MONIN): Resumen ejecutivo 2008-2009. Lima: INS; 2009.

12. Lohr SL. Sampling: design and analysis. California: Duxbury Press; 1999.

13. Pulgar Vidal J. Geografía del Perú. Las ocho regiones naturales del Perú. Lima: Editorial Universo; 1967.

14. Peru, Ministerio de Economía y Finanzas. Hacia la búsqueda de un nuevo instrumento de focalización para la asignación de recursos destinados a la inversión social adicional en el marco de la lucha contra la pobreza. Lima: MEF; 2001.

15. Instituto Nacional de Salud. Monitoreo Nacional de Indicadores Nutricionales (MONIN): Manual del encuestador bioquímico. Lima: INS; 2009.

16. Merck Laboratorios. 114670 Test Cloro en agua dulce y agua de mar: Indicaciones de uso. Darmstadt: Merck; 2007.

17. IDEXX Laboratories. How to use Colilert $囚$. Maine: IDEXX; 2007.

18. Merck Laboratorios. 101298 Readycult $₫$ coliformes 100. Darmstadt: Merck; 2008.

19. Rojas R. Guía para la vigilancia y control de la calidad del agua para consumo humano. Lima: CEPIS/OPS; 2002. 
20. SPSS Inc. SPSS Complex Samples 15.0. Chicago: SPSS; 2006.

21. Perú. Ministerio de Vivienda, Construcción y Saneamiento. Planes Nacionales de Saneamiento 20062015. Lima: MCVS; 2006.

22. Yauri F. Control de calidad de agua, Red Huaral y SBSUnidad de Epidemiología y Salud Ambiental. Lima: Ministerio de Salud; 2008.

23. Pérez-Cordón G, Rosales MJ, Valdez RA, VargasVásquez F, Cordova O. Detección de parásitos intestinales en agua y alimentos de Trujillo, Perú. Rev Peru Med Exp Salud Publica. 2008;25(1):144-48.

24. Oswald WE, Lescano AG, Bern C, Calderon MM, Cabrera L, Gilman RH. Fecal contamination of drinking water within peri-urban households, Lima, Peru. Am J Trop Med Hyg.2007;77(4):699-704.

25. Vergaray G, Mendez CR, Béjar VR, Morante HY, Heredia V. Coliformes injuriados en el agua de bebida de edificios de Lima-Cercado. Rev Inst Investig Fac Minas Metal Cienc Geogr. 2007;10(19):51-54.

26. García A, Pedreros L, Huapaya B. Vibrio cholerae $N^{\circ} \mathrm{O} 1$ en muestras de agua no cloradas consumidas por pobladores de las localidades de Santa y Coishco (Ancash), 2003-2004. Rev Peru Med Exp Salud Publica. 2006;23(3):168-72.

27. Besser RE, Moscoso B, Cabanillas O, Gonzalez L, Minaya P, Rodriguez $\mathbf{M}$, et al. Prevención de la transmisión del cólera: evaluación rápida de la calidad del agua municipal en Trujillo, Perú. Bol Oficina Sanit Panam. 1995;119(3):189-94
28. Perú, Instituto Nacional de Estadística e Informática. Censos Nacionales 2007, XI de Población y VI de Vivienda. 2007. Lima: INEl; 2008.

29. Prüs-Üstün A, Bos R, Gore F, Bartram J. Safe water, better health: cost, benefits and sustainability of interventions to protect and promote health. Geneva: WHO; 2008.

30. Clasen T, Roberts I, Rabie T, Schmidt W, Cairncross S. Interventions to improve water quality for preventing diarrhoea. Cochrane Database Syst Rev. 2006;3:CD004794.

31. Perú, Presidencia del Consejo de Ministros. Norma Técnica $N^{\circ}$ 001-2008 de Implementación regional y local de la Estrategia Nacional CRECER. Lima: Presidencia del Consejo de Ministros; 2008.

32. Bosch C, Hommann K, Sadoff C, Travers L. Agua, saneamiento y la pobreza. Borrador para Comentarios. Washington DC: Banco Mundial; 2001 Abr.

Correspondencia: Lic. Marianella Y. Miranda Cuadros Dirección: Tizon y Bueno 276, Jesus María, Lima11, Perú. Teléfono: (051) 617-6200 anexos 6605-6636.

Correo electrónico: mmiranda@ins.gob.pe

\section{Consulte las ediciones anteriores de la} Revista Peruana de Medicina Experimental y Salud Pública en www.scielo.org.pe

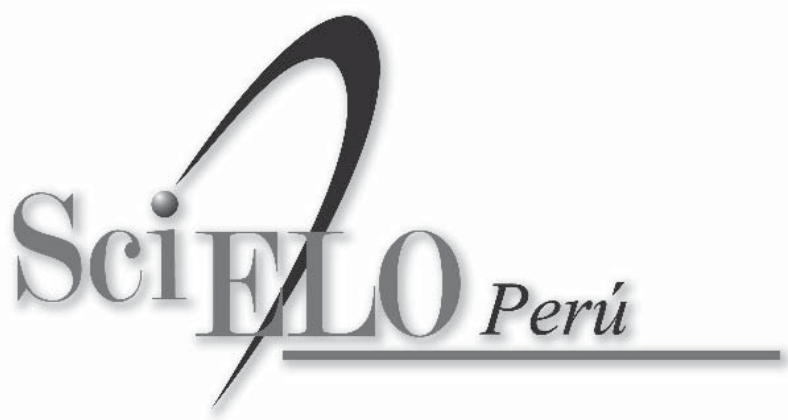

